\title{
Global attractor and asymptotic smoothing effects for the weakly damped cubic Schrödinger equation in $L^{2}(\mathbb{T})$
}

\author{
Luc Molinet
}

Communicated by Yvan Martel, received October 20, 2008.

\begin{abstract}
We prove that the weakly damped cubic Schrödinger flow in $L^{2}(\mathbb{T})$ provides a dynamical system that possesses a global attractor. The proof relies on a sharp study of the behavior of the associated flow-map with respect to the weak $L^{2}(\mathbb{T})$-convergence inspired by [18]. Combining the compactness in $L^{2}(\mathbb{T})$ of the attractor with the approach developed in $[\mathbf{1 0}]$, we show that the attractor is actually a compact set of $H^{2}(\mathbb{T})$. This asymptotic smoothing effect is optimal in view of the regularity of the steady states.
\end{abstract}

\section{Contents}

1. Introduction

2. Function spaces and notation

3. Proof of Theorem $1.3 \quad 18$

4. Existence of the global attractor 21

5. Asymptotic smoothing effect 22

References

\section{Introduction}

The cubic nonlinear Schrödinger equation (NLS) can be derived as an asymptotic model to describe long wave propagation in different dispersive media. In some physical contexts, an exterior forcing and damping effects have to be taken into account and this can lead to the following cubic NLS equation

$$
u_{t}+\gamma u+i u_{x x} \mp i|u|^{2} u=f,
$$

1991 Mathematics Subject Classification. 35

Key words and phrases. Global attractor, asymptotic smoothing, cubic Schrödinger flow.

The author is grateful to the Referee for several valuable remarks. The author was partially supported by the ANR project " Etude qualitative des EDP dispersives".

(c)2009 International Press 
where $\gamma>0$ is the damping parameter and $f$ is the forcing term. In this paper we focus on the case where $u(t, x)$ is a function from $\mathbb{R}_{+} \times \mathbb{T}$ to $\mathbb{C}$, with $\mathbb{T}=\mathbb{R} / 2 \pi \mathbb{Z}$, and $f \in L^{2}(\mathbb{T})$ does not depend on time. Also since the sign in front of the nonlinear term will not play any role in our analysis, we will take the + sign in all this paper.

It is well-known since the work of Bourgain [3] that (1) provides an infinite dimensional dynamical system on $H^{s}(\mathbb{T})$ for $s \geq 0$. Using an a priori estimate in $H^{1}(\mathbb{T})$ related to the energy conservation of the classical cubic NLS equation, the existence of a global attractor in $H^{1}(\mathbb{T})$ can be obtained by a standard method (see for instance $[\mathbf{2 2}]$ or $[\mathbf{9}]$ where the additional regularity of the attractor is also proved). This method principally contains two steps. A first step consists in proving the continuity of the flow-map associated with the equation with respect to the weak topology of the phase space. This ensures the existence of a compact global attractor for the weak topology. The second step uses the argument of Ball [2] to convert the weak convergence to the attractor into a strong one. The standard way to prove the first step is to use the well-posedness of the equation in a larger function space where the phase space is compactly embedded (cf. for instance $[6]$ ). This approach cannot be applied to $(1)$ in $L^{2}(\mathbb{T})$ since the well-posedness of this equation is not known in such a space. Actually, the strong ill-posedness of the classical cubic NLS equation in $H^{s}(\mathbb{T}), s<0$, has been even proved (cf. [5], [18]). In [12] Goubet and the author used another approach involving the so called Kato local smoothing effect for (1) on $\mathbb{R}$ to establish the weak continuity of the flow-map in $L^{2}(\mathbb{R})$. The situation in $L^{2}(\mathbb{T})$ seems more complicated since, as was shown in [18], the flowmap of the classical cubic NLS equation is discontinuous for the weak topology of $L^{2}(\mathbb{T})$. Note however that the result in [18] shows that the flow-map associated with the modified Schrödinger equation (see (16 below) introduced by Christ [4] is continuous for this weak topology. Let us mention here that the well-posedness of the modified NLS equation in a function space where $L^{2}(\mathbb{T})$ is continuously (but not compactly) embedded is obtained in [4] (see also [13] for a related result). Using results of $[\mathbf{1 8}]$, we clarify some behaviors of the flow-map of (1)with respect to the weak $L^{2}(\mathbb{T})$-convergence. From this information supplemented with the argument of Ball we deduce the existence of a global attractor in $L^{2}(\mathbb{T})$. Finally, combining the approach developed in $[\mathbf{9}]-[\mathbf{1 0}]$ with the compactness of the attractor, we prove that the global attractor actually belongs to $H^{2}(\mathbb{T})$ which can be viewed as an asymptotic smoothing effect. This smoothing effect is optimal since for $f$ belonging to $L^{2}(\mathbb{T})$ but not to $H^{s}(\mathbb{T})$, with $s>0$, the steady state to (1) does not belong to $H^{s}(\mathbb{T})$ for $s>2$.

Denoting by $S(\cdot)$ the nonlinear group associated with (1), i.e. $S(t) u_{0}:=$ $u(t), t \in \mathbb{R}$, where $u$ is the solution of (1) associated with the initial data $u_{0}$, our main result is as follows :

THEOREM 1.1. The nonlinear group $S(\cdot)$ associated with (1) provides an infinitedimensional dynamical system in $L^{2}(\mathbb{T})$ that has a global attractor $\mathcal{A}$ which is a compact set of $H^{2}(\mathbb{T})$. More precisely, $\mathcal{A}$ is a connected and compact set of $H^{2}(\mathbb{T})$, invariant (positively and negatively) by $S(\cdot)$ that attracts for the $L^{2}(\mathbb{T})$-metric all positive orbits uniformly with respect to bounded sets of initial data in $L^{2}(\mathbb{T})$.

REMARK 1.2. Exactly the same proof as in Section 5 below shows that the $L^{2}(\mathbb{R})$ global attractor to (1) on the line, that was constructed in $[\mathbf{1 2}]$, is actually a compact set of $H^{2}(\mathbb{R})$. 
The main new ingredient for proving Theorem 1.1 is the following result on the behavior of the flow-map of (1) with respect to the weak $L^{2}(\mathbb{T})$-convergence.

THEOREM 1.3. Let $\left\{u_{0, n}\right\}$ be a sequence of $L^{2}(\mathbb{T})$ converging weakly to $u_{0}$ in $L^{2}(\mathbb{T})$ and let $\left\{u_{n}\right\}$ be the sequence of the associated emanating solutions of the weakly damped cubic Schrödinger equation (1). For any adherence value $a_{0}$ of $\left\{\left\|u_{0, n}\right\|_{L^{2}}^{2}\right\}$ there exists a continuous function $t \mapsto a(t)$ from $\mathbb{R}$ to $\mathbb{R}_{+}$, with a $(0)=$ $a_{0}$, and a subsequence $\left\{u_{n_{k}}\right\}$ of $\left\{u_{n}\right\}$ such that, for any $t \in \mathbb{R}, u_{n_{k}}(t)$ converges weakly in $L^{2}(\mathbb{T})$ to $v(t)$ where $v \in C\left(\mathbb{R} ; L^{2}(\mathbb{T})\right) \cap L_{\text {loc }}^{4}(\mathbb{R} \times \mathbb{T})$ is the unique solution to

$$
\left\{\begin{array}{l}
v_{t}+i v_{x x}+\gamma v+i|v|^{2} v+\frac{i}{\pi}\left(a(\cdot)-\|v(\cdot)\|_{L^{2}}^{2}\right) v=f \\
v(0)=u_{0}
\end{array} .\right.
$$

REMARK 1.4. It is worth noticing that this theorem ensures that, in sharp contrast with the case on the line (cf. [12]), the flow-map associated with (1) is not continuous for the weak topology of $L^{2}(\mathbb{T})$. Indeed, following [18], let $u_{0} \in L^{2}(\mathbb{T})$ be different from 0 and let $\left\{\phi_{n}\right\} \subset L^{2}(\mathbb{T})$ be a sequence such that $\phi_{n} \rightarrow 0$ in $L^{2}(\mathbb{T})$ and $\left\|\phi_{n}\right\|_{L^{2}}^{2} \rightarrow 2 \pi$ as $n$ goes to infinity (one can take for instance $\phi_{n}=e^{i n x}$ ). Setting $u_{0, n}=u_{0}+\phi_{n}$, we get that $u_{0, n} \rightarrow u_{0}$ in $L^{2}(\mathbb{T})$ and $\left\|u_{0, n}\right\|_{L^{2}}^{2} \rightarrow\left\|u_{0}\right\|_{L^{2}}^{2}+2 \pi$ as $n \rightarrow \infty$. On account of Theorem 1.3, the emanating solutions $u_{n}$ tend weakly in $L^{2}(\mathbb{T})$ for any fixed $t \in \mathbb{R}$ to $v$ satisfying (2). Observe that $w=v-u$ is solution of

$$
\left\{\begin{array}{l}
w_{t}+i w_{x x}+\gamma w+i\left(|v|^{2} w+(\bar{w} u+w \bar{v}) u\right)=-\frac{i}{\pi}\left(a(\cdot)-\|v(\cdot)\|_{L^{2}}^{2}\right) v . \\
w(0)=0
\end{array} .\right.
$$

Since $v(0)=u_{0} \neq 0$ and $a(0)=\left\|u_{0}\right\|_{L^{2}}^{2}+2 \pi \neq\|v(0)\|_{L^{2}}^{2}$ we infer that the $L^{2}(\mathbb{T})$ norm of the right-hand side of (3) cannot vanish for small $t \neq 0$. Hence $w(t)=0$ is not a solution of (3) and thus $v(t) \neq u(t)$ for small $t \neq 0$.

Finally, note that, since $L^{2}(\mathbb{T})$ is compactly embedded in $H^{s}(\mathbb{T})$ for $s<0$, this proves that (1) is ill-posed in $H^{s}(\mathbb{T})$ as soon as $s<0$.

This paper is organized as follows. In the next section we introduce some notation and the function spaces we will work with. Section 3 is devoted to the proof of Theorem 1.3 and Section 4 is devoted to the existence of the global attractor. Finally in Section 5 we prove the asymptotic smoothing effect.

\section{Function spaces and notation}

When we affirm that a proposition is valid for $x+$ (respectively $x-$ ) with $x \in \mathbb{R}$, we mean that there exists a small real number $\epsilon>0$ such that the proposition is valid for any real number in the interval $] x, x+\epsilon[$ (respectively $] x-\epsilon, x[$ ). For $(x, y) \in \mathbb{R}^{2}, x \lesssim y$ means that there exists $C>0$ such that $x \leq C y$. We will also denote by $\varepsilon$ any function from $\mathbb{R}_{+}$into itself that goes to zero at infinity.

For a $2 \pi$-periodic function $\varphi$, we define its space Fourier transform by

$$
\hat{\varphi}(k):=\frac{1}{2 \pi} \int_{\mathbb{T}} e^{-i k x} \varphi(x) d x, \quad \forall k \in \mathbb{Z},
$$

and we denote by $P_{N} \varphi$ and $Q_{N} \varphi$ the $L^{2}(\mathbb{T})$ orthogonal projections on respectively the space Fourier modes $|k| \leq N$ and $|k|>N$. 
We denote by $V(\cdot)$ the free group associated with the linearized Schrödinger equation,

$$
\widehat{V(t) \varphi}(k):=e^{-i k^{2} t} \hat{\varphi}(k), \quad k \in \mathbb{Z} .
$$

The Sobolev spaces $H^{s}(\mathbb{T})$ for $2 \pi$-periodic functions are defined as usually and endowed with

$$
\|\varphi\|_{H^{s}(\mathbb{T})}:=\left\|\langle k\rangle^{s} \widehat{\varphi}(k)\right\|_{l^{2}(\mathbb{Z})}=\left\|J_{x}^{s} \varphi\right\|_{L^{2}(\mathbb{T})},
$$

where $\langle\cdot\rangle:=\left(1+|\cdot|^{2}\right)^{1 / 2}$ and $\widehat{J_{x}^{s} \varphi}(k):=\langle k\rangle^{s} \widehat{\varphi}(k)$.

For a function $u(t, x)$ on $\mathbb{R} \times \mathbb{T}$, we define its space-time Fourier transform by

$$
\hat{u}(\tau, \xi):=\mathcal{F}_{t, x}(u)(\tau, \xi):=\frac{1}{2 \pi} \int_{\mathbb{R} \times \mathbb{T}} e^{-i(\tau t+k x)} u(t, x) d t d x, \quad \forall(\tau, k) \in \mathbb{R} \times \mathbb{Z} .
$$

and define the Bourgain spaces $X^{b, s}$ and $\tilde{X}^{b, s}$ of functions on $\mathbb{R} \times \mathbb{T}$ respectively endowed with the norm

$$
\|u\|_{X^{b, s}}:=\left\|\left\langle\tau+k^{2}\right\rangle^{b}\langle k\rangle^{s} \hat{u}\right\|_{L^{2}\left(\mathbb{R} ; l^{2}(\mathbb{Z})\right)}=\left\|\langle\tau\rangle^{b}\langle k\rangle^{s} \mathcal{F}_{t, x}(V(-t) u)\right\|_{L^{2}\left(\mathbb{R} ; l^{2}(\mathbb{Z})\right)} .
$$

and

$$
\|u\|_{\tilde{X}^{b, s}}:=\left\|\left\langle\tau-k^{2}\right\rangle^{b}\langle k\rangle^{s} \hat{u}\right\|_{L^{2}\left(\mathbb{R} ; l^{2}(\mathbb{Z})\right)}=\left\|\langle\tau\rangle^{b}\langle k\rangle^{s} \mathcal{F}_{t, x}(V(t) u)\right\|_{L^{2}\left(\mathbb{R} ; l^{2}(\mathbb{Z})\right)} .
$$

Finally, for an open interval $I \subset \mathbb{R}$ we define the restriction in time spaces $X_{I}^{b, s}$ of functions on $I \times \mathbb{T}$ endowed with the norm

$$
\|u\|_{X_{I}^{b, s}}:=\inf _{v \in X^{b, s}}\left\{\|v\|_{X^{b, s}}, v(\cdot) \equiv u(\cdot) \text { on } I\right\} .
$$

It is worth noticing that the $X_{I}^{b, s}$ spaces are Hilbert spaces with dual (for the $L^{2}$-duality) $X_{I}^{-b,-s}$ and that for any $\theta \in[0,1]$ it holds

$$
X_{I}^{\theta b_{1}+(1-\theta) b_{2}, s}=\left[X_{I}^{b_{1}, s}, X_{I}^{b_{2}, s}\right]^{\theta} .
$$

Moreover, for $b>1 / 2, X_{I}^{b, s}$ is continuously embedding in $L^{\infty}\left(I ; H^{s}(\mathbb{T})\right)$ with a constant of continuity that depends on $b$ and on $|I|$ the length of $I$, i.e.

$$
\|u\|_{L^{\infty}\left(I ; H^{s}(\mathbb{T})\right)} \leq C(b,|I|)\|u\|_{X_{I}^{b, s}}, \forall u \in X_{I}^{b, s}
$$

\section{Proof of Theorem 1.3}

Theorem 1.3 is based on the observation made in $[\mathbf{1 8}]$ on the cubic NLS equation posed on the one-dimensional torus. We first recall the following well-posedness result due to Bourgain $([\mathbf{3}])$ for $(1)$. Let us mention that this result was established for the cubic Schrödinger equation without damping and forcing but the adaptations for (1) are straightforward.

TheOREM 3.1. Let $s \geq 0$. For any $u_{0} \in H^{s}(\mathbb{T}), f \in H^{s}(\mathbb{T})$ and any $T>0$, there exists a unique solution

$$
u \in L^{4}(]-T, T[\times \mathbb{T})
$$

satisfying (1) in $\mathcal{D}^{\prime}(]-T, T[\times \mathbb{T})$. Moreover $u \in C\left([-T, T] ; H^{s}(\mathbb{T})\right) \cap X_{]-T, T[}^{1 / 2+, s}$ and the map data to solution $u_{0} \mapsto u$ is real analytic from $H^{s}(\mathbb{T})$ to $C\left([-T, T] ; H^{s}(\mathbb{T})\right)$. 
Let us recall that this theorem principally use the linear estimates in Bourgain's spaces for the free evolution and the retarded Duhamel operator

$$
\|V(t) \varphi\|_{X_{]-T, T[}^{b, s}} \leq C(T, b)\|\varphi\|_{H^{s}}, b \in \mathbb{R}, s \in \mathbb{R}, 0<T<1,
$$

and for any $0<\varepsilon<<1$ and $0<T<1$,

$$
\left\|\int_{0}^{t} V\left(t-t^{\prime}\right) g\left(t^{\prime}\right) d t^{\prime}\right\|_{X_{]-T, T[}^{b, s}} \leq C(b, \varepsilon) T^{\varepsilon}\|g\|_{X_{]-T, T[}^{b-1+\varepsilon, s}}, 1 / 2 \leq b<1,
$$

as well as the following linear dispersive estimate

$$
\|v\|_{L^{4}(\mathbb{R} \times \mathbb{T})} \lesssim\|v\|_{X^{3 / 8,0}}, \quad \forall v \in X^{3 / 8,0}
$$

This estimate is proven in $[\mathbf{3}]$ for functions on $\mathbb{T}^{2}$ but also holds for functions on $\mathbb{R} \times \mathbb{T}$ (See $[\mathbf{1 7}]$ for a shorter proof that works also clearly on $\mathbb{R} \times \mathbb{T}$ ). Moreover, according to $[\boldsymbol{7}],(7)$ ensures that for $0<T<1$ it holds

$$
\|V(t) \varphi\|_{L^{4}(]-T, T[\times \mathbb{T})} \lesssim T^{1 / 8}\|\varphi\|_{L^{2}(\mathbb{T})}, \quad \forall \varphi \in L^{2}(\mathbb{T}),
$$

which gives directly the existence and uniqueness in $L^{4}(]-T, T[\times \mathbb{T})$ by classical $T T^{*}$ arguments. On the other hand, to prove that $u \in X_{]-T, T[}^{1 / 2+, 0}$ one has to notice that, applying (7) with $\bar{v},(7)$ clearly also holds with $X^{3 / 8,0}$ replaced by $\tilde{X}^{3 / 8,0}$. Therefore,

$$
\left\|\mathcal{F}^{-1}(|\widehat{\bar{v}}|)\right\|_{L^{4}\left(\mathbb{T}^{2}\right)} \lesssim\|\bar{v}\|_{\tilde{X}^{3 / 8,0}}=\|v\|_{X^{3 / 8,0}}, \quad \forall v \in X^{3 / 8,0}
$$

and (7)-(9) yield

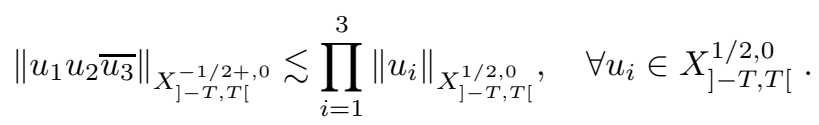

Writing the Duhamel formulation of (1), using (5)-(6) and (10) and choosing some small positive real number $\varepsilon$, one can eventually derive the key estimate:

$$
\|u\|_{X_{]-T, T[}^{1 / 2+, 0}} \lesssim\left\|u_{0}\right\|_{L^{2}(\mathbb{T})}+T^{0+}\left[\left(\|u\|_{X_{]-T, T[}^{1 / 2+, 0}}^{2}+\gamma\right)\|u\|_{X_{]-T, T[}^{1 / 2+, 0}}+\|f\|_{L^{2}(\mathbb{T})}\right] .
$$

This leads to the local existence result in $X_{]-T, T[}^{1 / 2+, 0}$. Finally, the fact that the time of existence in Theorem 3.1 can be chosen arbitrarly large follows from the a priori bound on the $L^{2}(\mathbb{T})$-norm of the solution (see (13)-(14) below).

Now, let $u_{0} \in L^{2}(\mathbb{T})$ and $\left\{u_{0, n}\right\} \subset L^{2}(\mathbb{T})$ be a sequence converging weakly to $u_{0}$ in $L^{2}(\mathbb{T})$. Note that, from Banach Steinhaus'theorem, $\left\{\left\|u_{0, n}\right\|_{L^{2}(\mathbb{T})}\right\}$ is bounded in $\mathbb{R}_{+}$. It is well-known that the solutions of (1), given by Theorem 3.1 , satisfy for all $t \in \mathbb{R}$,

$$
\frac{1}{2} \frac{d}{d t}\|u\|_{L^{2}(\mathbb{T})}^{2}+\gamma\|u\|_{L^{2}(\mathbb{T})}^{2}=\operatorname{Re} \int_{\mathbb{T}} f \bar{u} d x
$$

By Young's inequality and Gronwall's lemma, we deduce that the $L^{2}$-solutions satisfy for any $t \in \mathbb{R}_{+}$,

$$
\|u(t)\|_{L^{2}(\mathbb{T})}^{2} \leq e^{-\gamma t}\left\|u_{0}\right\|_{L^{2}(\mathbb{T})}^{2}+\frac{1-e^{-\gamma t}}{\gamma^{2}}\|f\|_{L^{2}(\mathbb{T})}^{2} .
$$


Performing the change of variables $(t, x) \mapsto(-t, x)$ and proceeding as above we also infer that for any $t \in \mathbb{R}_{-}$it holds

$$
\|u(t)\|_{L^{2}(\mathbb{T})}^{2} \leq e^{3 \gamma|t|}\left\|u_{0}\right\|_{L^{2}(\mathbb{T})}^{2}+\frac{e^{3 \gamma|t|}-1}{\gamma^{2}}\|f\|_{L^{2}(\mathbb{T})}^{2}
$$

Therefore, from (12), we deduce that for any $\left(t_{0}, t_{1}\right) \in \mathbb{R}^{2}$ with $t_{1}>t_{0}$,

$$
\begin{aligned}
\left|\left\|u\left(t_{1}\right)\right\|_{L_{x}^{2}}^{2}-\left\|u\left(t_{0}\right)\right\|_{L_{x}^{2}}^{2}\right|= & \left|2 \gamma \int_{t_{0}}^{t_{1}}\|u(\tau)\|_{L^{2}(\mathbb{T})}^{2} d \tau+2 \operatorname{Re} \int_{t_{0}}^{t_{1}} \int_{\mathbb{T}} f \bar{u}(\tau) d x d \tau\right| \\
\leq & \left|t_{1}-t_{0}\right|\left[3 \gamma\left(e^{3 \gamma\left|t_{1}\right|}\left\|u_{0}\right\|_{L^{2}(\mathbb{T})}^{2}+\frac{e^{3 \gamma\left|t_{1}\right|}-1}{\gamma^{2}}\|f\|_{L^{2}(\mathbb{T})}^{2}\right)\right. \\
& \left.+\frac{1}{\gamma}\|f\|_{L^{2}(\mathbb{T})}^{2}\right] .
\end{aligned}
$$

Denoting by $u_{n}$ the solution to (1) associated with the initial data $u_{0, n}$, this last inequality ensures that the sequence $\left\{t \mapsto\left\|u_{n}(t)\right\|_{L^{2}(\mathbb{T})}^{2}\right\}$ is uniformly equi-continuous on any bounded interval of $\mathbb{R}$. It follows from Ascoli's theorem that there exists a subsequence $\left\{t \mapsto\left\|u_{n_{k}}(t)\right\|_{L^{2}(\mathbb{T})}^{2}\right\}$ that converges to some function $t \mapsto a(t)$ in $C\left([-T, T] ; \mathbb{R}_{+}\right)$for any $T>0$. Moreover, from Theorem 3.1 we know that $\left\{u_{n_{k}}\right\}$ is bounded in $X_{]-T, T[}^{1 / 2+, 0}$ and thus, up to the extraction of a subsequence, converges weakly to some $v$ in $X_{]-T, T[}^{1 / 2+, 0}$.

Now, in $([\mathbf{1 8}]$, Lemmas $2 \& 3$ ) it is proven that the nonlinear term of the modified Schrödinger equation introduced in [4]:

$$
\Lambda(u):=|u|^{2} u-\frac{1}{\pi}\|u\|_{L^{2}}^{2} u
$$

is continuous from $\left(X_{1}^{1 / 2+, 0}\right)^{3}$ into $X_{1}^{-7 / 16,0}$ equipped with their respective weak topology. We thus rewrite the Duhamel formulation for $u_{n}$ in the following way :

$$
\begin{aligned}
u_{n}(t)= & V(t) u_{0, n}-i \int_{0}^{t} V\left(t-t^{\prime}\right) \Lambda\left(u_{n}\left(t^{\prime}\right)\right) d t^{\prime} \\
& -\frac{i}{\pi} \int_{0}^{t} V\left(t-t^{\prime}\right)\left(\left\|u_{n}\left(t^{\prime}\right)\right\|_{L^{2}}^{2} u_{n}\left(t^{\prime}\right)\right) d t^{\prime}-\gamma \int_{0}^{t} V\left(t-t^{\prime}\right) u_{n}\left(t^{\prime}\right) d t^{\prime} \\
& +\int_{0}^{t} V\left(t-t^{\prime}\right) f d t^{\prime}
\end{aligned}
$$

Since $u_{n_{k}} \rightarrow v$ in $X_{]-T, T[}^{1 / 2+, 0} \hookrightarrow C\left([-T, T] ; L^{2}(\mathbb{T})\right)$ and $a_{n_{k}}(\cdot) \rightarrow a(\cdot)$ in $C\left([-T, T] ; L^{2}(\mathbb{T})\right)$, it follows that $a_{n_{k}}(\cdot) u_{n_{k}} \rightarrow a(\cdot) v$ in $C\left([-T, T] ; L^{2}(\mathbb{T})\right)$. According to the continuity of the Duhamel operator from $C\left([-T, T] ; L^{2}(\mathbb{T})\right)$ into itself, the linear estimates (5)-(6), the continuity result on $\Lambda$ for the weak topology and the above convergence results, we can pass to the limit to obtain that

$$
\begin{aligned}
v(t) & =V(t) u_{0}-i \int_{0}^{t} V\left(t-t^{\prime}\right) \Lambda\left(v\left(t^{\prime}\right)\right) d t^{\prime} \\
-\frac{i}{\pi} & \int_{0}^{t} V\left(t-t^{\prime}\right)\left(a\left(t^{\prime}\right) v\left(t^{\prime}\right)\right) d t^{\prime}-\gamma \int_{0}^{t} V\left(t-t^{\prime}\right) v\left(t^{\prime}\right) d t^{\prime}+\int_{0}^{t} V\left(t-t^{\prime}\right) f d t^{\prime}
\end{aligned}
$$


and $v$ is solution of the following Cauchy problem on $]-T, T[$ :

$$
\left\{\begin{array}{l}
v_{t}+v_{x x}+\gamma v+i \Lambda(v)+\frac{i}{\pi} a(\cdot) v=f \text { in } \mathcal{D}^{\prime}(]-T, T[\times \mathbb{T}) \\
v(0)=u_{0}
\end{array} .\right.
$$

Proceeding exactly as for the cubic Schrödinger equation, it is easy to prove that this Cauchy problem is globally well-posed ${ }^{1}$ in $H^{s}(\mathbb{T}), s \geq 0$, with a solution belonging for all $T>0$ to

$$
C\left([-T, T] ; H^{s}(\mathbb{T})\right) \cap L^{4}(]-T, T[\times \mathbb{T})
$$

with uniqueness in $L^{4}(]-T, T[\times \mathbb{T})$. Therefore, there exists only one possible limit and thus the sequence $\left\{u_{n_{k}}\right\}$, and not only a subsequence of it, converges weakly to $v$ in $X_{]-1,1[}^{1 / 2+, 0}$. Moreover, using the equation satisfied by the $u_{n}$ and the uniform bound in $L^{\infty}(]-T, T\left[; L^{2}(\mathbb{T})\right) \cap L^{4}(]-T, T[\times \mathbb{T})$, it is easy to check that for any smooth $2 \pi$-periodic function $\phi$, the family $\left\{t \mapsto\left(u_{n_{k}}(t), \phi\right)_{L^{2}}\right\}$ is bounded in $C([-1,1])$ and uniformly equi-continuous on $[-1,1]$. Ascoli's theorem then ensures that $\left(u_{n_{k}}, \phi\right)$ converges to $(v, \phi)$ on $[-1,1]$ and thus $u_{n_{k}}(t) \rightarrow v(t)$ in $L^{2}(\mathbb{T})$ for all $t \in[-1,1]$. By direct iteration this clearly also holds for all $t \in \mathbb{R}$.

Finally, according to (16), $v$ can be also characterized as the unique solution in $L^{4}(]-T, T[\times \mathbb{T})$ to

$$
\left\{\begin{array}{l}
v_{t}+i v_{x x}+\gamma v+i|v|^{2} v+\frac{i}{\pi}\left(a(\cdot)-\|v(\cdot)\|_{L^{2}}^{2}\right) v=f \\
v(0)=u_{0}
\end{array} .\right.
$$

\section{Existence of the global attractor}

Let us denote by $S(t)$ the nonlinear group associated with (1), i.e.

$$
S(t) u_{0}:=u(t), t \in \mathbb{R} .
$$

On account of Theorem 1.3 and (13), we infer that the ball of $L^{2}(\mathbb{T})$,

$$
X:=\left\{v \in L^{2}(\mathbb{T}),\|v\|_{L^{2}(\mathbb{T})} \leq M_{0}:=2 \frac{\|f\|_{L^{2}(\mathbb{T})}}{\gamma}\right\}
$$

is a global absorbing set for the dynamical system under consideration and that $S(t)$ acts continuously on $X$. To prove that there exists a global attractor it suffices to check the relative compactness in $L^{2}(\mathbb{T})$ of sequences of the type $\left\{S\left(t_{n}\right) b_{n}\right\}$ with $t_{n} \uparrow+\infty$ and $\left\{b_{n}\right\} \subset X$. This is the aim of the following proposition.

Proposition 4.1. For any sequences $\left\{b_{n}\right\} \subset X$ and $\left\{t_{n}\right\} \uparrow+\infty$, the sequence $\left\{S\left(t_{n}\right) b_{n}\right\}$ has an adherence value in $L^{2}(\mathbb{T})$.

Proof. We combine Theorem 1.3 with the famous J. Ball's argument (see [2], [22], [16]). Let $\left\{b_{n}\right\} \subset X$ and let $\left\{t_{n}\right\}$ be a sequence of positive real numbers that goes to infinity. From (13) the sequence $\left\{S\left(t_{n}\right) b_{n}\right\}$ remains bounded in $L^{2}(\mathbb{T})$ and thus, up to the extraction of a subsequence, converges weakly in $L^{2}(\mathbb{T})$ to some $v_{0}$. According to Theorem 1.3 there exists a subsequence $\left\{S\left(t_{n_{k}}\right) b_{n_{k}}\right\}$ and a continuous function $t \mapsto a(t)$ from $\mathbb{R}$ to $\mathbb{R}_{+}$such that the solutions emanating from $\left\{S\left(t_{n_{k}} b_{n_{k}}\right)\right\}$ converge weakly in $L^{2}(\mathbb{T})$ for all $t \in \mathbb{R}$ to $v(t)$ where $v$ is the unique solution to

$$
\left\{\begin{array}{l}
v_{t}+i v_{x x}+\gamma v+i|v|^{2} v+\frac{i}{\pi}\left(a(\cdot)-\|v(\cdot)\|_{L^{2}}^{2}\right) v=f \\
v(0)=v_{0}
\end{array} .\right.
$$

\footnotetext{
${ }^{1}$ Note that the $L^{2}$-norm is controlled on any bounded interval of $\mathbb{R}$
} 
From (12) we infer that for $\tau>0$ fixed and $n_{k}$ large enough,

$\left\|S\left(t_{n_{k}}\right) b_{n_{k}}\right\|_{L^{2}(\mathbb{T})}^{2}=e^{-2 \gamma \tau}\left\|S\left(t_{n_{k}}-\tau\right) b_{n_{k}}\right\|_{L^{2}(\mathbb{T})}^{2}-2 \operatorname{Re} \int_{0}^{\tau} \int_{\mathbb{T}} e^{-2 \gamma s} \bar{f} S\left(t_{n_{k}}-s\right) b_{n_{k}} d s d x$

where $\left\|S\left(t_{n_{k}}-\tau\right) b_{n_{k}}\right\|_{L^{2}(\mathbb{T})}^{2} \leq M_{0}^{2}$ and, according to the weak convergence and the dominated convergence theorem,

(22) $\lim _{n_{k} \rightarrow+\infty} 2 \operatorname{Re} \int_{0}^{\tau} \int_{\mathbb{T}} e^{-2 \gamma s} \bar{f} S\left(t_{n_{k}}-s\right) b_{n_{k}} d x d s=2 \operatorname{Re} \int_{0}^{\tau} \int_{\mathbb{T}} e^{-2 \gamma s} \bar{f} v(-s) d x d s$.

On the other hand, using the energy identity for equation (20), we get

$$
\left\|v_{0}\right\|_{L^{2}(\mathbb{T})}^{2}=e^{-2 \gamma \tau}\|v(-\tau)\|_{L^{2}(\mathbb{T})}^{2}-2 \operatorname{Re} \int_{0}^{\tau} \int_{\mathbb{T}} e^{-2 \gamma s} \bar{f} v(-s) d x d s .
$$

But since $S\left(t_{n_{k}}-\tau\right) b_{n_{k}} \rightarrow v(-\tau)$ in $L^{2}(\mathbb{T})$, it follows from (13) that

$$
\|v(-\tau)\|_{L^{2}(\mathbb{T})}^{2} \leq M_{0}^{2}
$$

Gathering the above three equalities, we thus infer that for any fixed $\tau>0$,

$$
\limsup _{n_{k} \rightarrow+\infty}\left\|S\left(t_{n_{k}}\right) b_{n_{k}}\right\|_{L^{2}(\mathbb{T})}^{2} \leq\left\|v_{0}\right\|_{L^{2}(\mathbb{T})}^{2}+2 e^{-2 \gamma \tau} M_{0}^{2}
$$

which ensures that $S\left(t_{n_{k}}\right) b_{n_{k}}$ converges actually strongly to $v_{0}$ in $L^{2}(\mathbb{T})$. This completes the proof of Proposition 4.1.

Proposition 4.1 ensures the existence of a compact global attractor in $L^{2}(\mathbb{T})$. More precisely, from classical arguments (see for instance the proof of Theorem 1.1 in $[\mathbf{2 0}])$, it follows that the positively invariant connected closed set

$$
\mathcal{A}:=\omega(X)=\bigcap_{s>0} \overline{\bigcup_{t>s} S(t) X}
$$

is non-empty and attracts any bounded set of $L^{2}(\mathbb{T})$. The compactness of $\mathcal{A}$ follows as well. Indeed, let $\left\{a_{n}\right\} \subset \mathcal{A}$. Taking a sequence $\left\{t_{n}\right\} \uparrow+\infty$ and setting $b_{n}=$ $S\left(-t_{n}\right) a_{n}$, we get that $a_{n}=S\left(t_{n}\right) b_{n}$ with $\left\{b_{n}\right\} \subset \mathcal{A} \subset X$ and thus $\left\{a_{n}\right\}$ has got an adherence point in $L^{2}(\mathbb{T})$. Finally, it is worth noticing that, by construction, $\mathcal{A}$ is also negatively invariant.

\section{Asymptotic smoothing effect}

In this section we prove that the global attractor lies actually in $H^{2}(\mathbb{T})$ and is moreover compact in this space. Following the approach developed in [10], we split the solution $u(t)=S(t) u_{0}$ emanating from $u_{0}$ into two parts by setting ${ }^{2}$

$$
\begin{gathered}
v_{t}+i v_{x x}+\gamma v+i|v|^{2} v=f-i P_{N}\left(|u|^{2} u\right)+i P_{N}\left(|v|^{2} v\right) \\
w_{t}+i w_{x x}+\gamma w=-i Q_{N}\left(|w|^{2} w-2|w|^{2} u-w^{2} \bar{u}\right)-i Q_{N}\left(2|u|^{2} w+u^{2} \bar{w}\right)
\end{gathered}
$$

with initial conditions

$$
v(0)=P_{N}\left(u_{0}\right) \text { and } w(0)=Q_{N}\left(u_{0}\right) .
$$

\footnotetext{
${ }^{2}$ Recall that $P_{N}$ and $Q_{N}$ are the projections on respectively the spatial Fourier modes $|k| \leq N$ and $|k|>N$.
} 
REMARK 5.1. Proceeding as for the equation (1) it is easy to check that, $u \in$ $X_{T}^{1 / 2+, 0}$ and $f \in L^{2}(\mathbb{T})$ being given, the Cauchy problems (25) and (26) are locally well-posed in $L^{2}(\mathbb{T})$. Hence, there exists $\alpha>0$ and a unique solution $v \in X_{]-\alpha, \alpha[}^{1 / 2+, 0}$ of (25) and $w \in X_{]-\alpha, \alpha[}^{1 / 2+, 0}$ of (26). Actually we will see in this section that $w \in$ $C\left(\mathbb{R}_{+} ; L^{2}(\mathbb{T})\right)$ and $v \in C\left(\mathbb{R}_{+} ; H^{2}(\mathbb{T})\right)$.

In [10], Goubet introduced this decomposition for the weakly damped KdV equation. A first step of his analysis consists in proving that the high frequency part $w(t)$ is decreasing to 0 in $L^{2}(\mathbb{T})$. This decay of $\|w(t)\|_{L^{2}(\mathbb{T})}$, which is uniform for all $u_{0}$ in the absorbing ball, is obtained by using the dispersive damping effect on the high-high frequencies interactions that occurs for the nonlinear part of the $\mathrm{KdV}$ equation above $H^{-1 / 2}(\mathbb{T})$. This is related to the fact that the associated Cauchy problem is well-posed in $H^{s}(\mathbb{T})$ for $s \geq-1 / 2$. For the cubic Schrödinger equation the situation is more delicate since as recalled in the introduction this equation is ill-posed in $H^{s}(\mathbb{T})$ for $s<0$. Actually, due to some resonant parts in the nonlinear term, there is no damping effect on high-high-high interactions. To overcome this difficulty we will work directly on the global attractor and use in a crucial way that we already proved that it is compact in $L^{2}(\mathbb{T})$. Note that the a priori compactness of the global attractor is not required in $[\mathbf{1 0}]$ where the compactness of the attractor can be obtained as a consequence of the asymptotic behavior of $v$ and $w$.

The second step of the analysis in [10] consists in proving an uniform bound in $H^{3}(\mathbb{T})$ on $v$. This uniform estimate follows from an uniform bound in $L^{2}(\mathbb{T})$ on the time derivative $v_{t}$ of $v$. To get this last bound the author uses that, in view of the equation satisfied by $v$, the low frequencies $P_{N} v_{t}$ belongs to any $H^{s}(\mathbb{T}), s \in \mathbb{R}$. We will not be able to use this approach here since for $v \in L^{2}(\mathbb{T}), P_{N}\left(|v|^{2} v\right)$ does not belong a priori to any $H^{s}(\mathbb{T})$. Inspired by $[\mathbf{2 1}]$ we will instead introduce the auxiliary function $z:=Q_{N}(v-g)$, where $g$ is defined by $\widehat{g}(k):=\frac{\widehat{f}(k)}{-i k^{2}+\gamma}$, and prove that $z$ is uniformly bounded in $H^{2}(\mathbb{T})$.

The key proposition to derive the regularity of the attractor is the following.

Proposition 5.2. There exist functions $h$ and $K: \mathbb{R}_{+} \rightarrow \mathbb{R}_{+}$with $\lim _{t \rightarrow+\infty} h(t)=$ 0 such that for all $N>0$ large enough and all $u_{0} \in \mathcal{A}$ the function $v$ and $w$ constructed in (25)-(26) satisfy

$$
\|w(t)\|_{L^{2}(\mathbb{T})} \leq h(t) \text { and }\|v(t)\|_{H^{2}(\mathbb{T})} \leq K(N), \forall t \in \mathbb{R}_{+} .
$$

With Proposition 5.2 at hand it is straightforward to check that $\mathcal{A}$ is embedded in $H^{2}(\mathbb{T})$. Indeed, let $a \in \mathcal{A}$ and $\left\{t_{n}\right\} \uparrow+\infty$. For all $n \in \mathbb{N}$ we can write $a$ as

$$
a=S\left(t_{n}\right) S\left(-t_{n}\right) a=S\left(t_{n}\right) b_{n}
$$

with $b_{n}=S\left(-t_{n}\right) a \in \mathcal{A}$. From Proposition 5.2 it follows that, for any $n \in \mathbb{N}, a$ can be decomposed as $a=v_{n}+w_{n}$ with $\left\|v_{n}\right\|_{H^{2}(\mathbb{T})} \leq K$ and $\left\|w_{n}\right\|_{L^{2}(\mathbb{T})} \rightarrow 0$ as $n \rightarrow+\infty$. Therefore $a \in H^{2}(\mathbb{T})$ and $\|a\|_{H^{2}(\mathbb{T})} \leq K$. Hence, there exists $K>0$, such that the following uniform bound holds on the attractor :

$$
\|a\|_{H^{2}(\mathbb{T})} \leq K, \forall a \in \mathcal{A} .
$$

\subsection{Proof of Proposition 5.2.}


5.1.1. Preliminaries. The $L^{2}(\mathbb{T})$-compactness of $\mathcal{A}$ ensures the following uniform bound on the $L^{2}(\mathbb{T})$-norm of the high frequency part to the elements of $\mathcal{A}$.

Proposition 5.3. There exists a function $\varepsilon$ from $\mathbb{R}_{+}$into itself that goes to zero at infinity such that

$$
\left\|Q_{N} a\right\|_{L^{2}(\mathbb{T})} \leq \varepsilon(N), \forall a \in \mathcal{A}
$$

Thanks to this remark we will have to prove a damping effect only on terms of the form $P_{N / 2} u_{1} P_{N / 2} u_{2} \overline{Q_{N} u_{3}}$. This is the aim of the following lemma :

Lemma 5.4. Let $I \subset \mathbb{R}$ be a bounded interval and let $u_{i} \in X_{I}^{1 / 2,0}, i=1,2,3$. Then for $\epsilon>0$ small enough it holds

$$
\left\|P_{N / 2} u_{1} P_{N / 2} u_{2} \overline{Q_{N} u_{3}}\right\|_{X_{I}^{-1 / 2+\epsilon, 0}} \lesssim N^{-1 / 4+2 \epsilon} \prod_{i=1}^{3}\left\|u_{i}\right\|_{X_{I}^{1 / 2,0}} .
$$

Proof. We take extensions $v_{i}$ of the $u_{i}$ 's such that $\left\|v_{i}\right\|_{X^{1 / 2,0}} \leq 2\left\|u_{i}\right\|_{X_{I}^{1 / 2,0}}$. By duality we have to prove that

$$
\left.\sup _{\|w\|_{X^{1 / 2-\epsilon, 0}=1}} \mid\left(w, P_{N / 2} v_{1} P_{N / 2} v_{2} \overline{Q_{N} v_{3}}\right)\right)_{L^{2}(\mathbb{R} \times \mathbb{T})} \mid \lesssim N^{-1 / 4+2 \epsilon} \prod_{i=1}^{3}\left\|v_{i}\right\|_{X^{1 / 2,0}} .
$$

It thus suffices to estimate

$$
J=\int_{\mathbb{R}^{3}} \sum_{\left(k_{1}, k_{2}, k_{3}\right) \in A(N)}|\widehat{w}(\tau, k)|\left|\widehat{v_{1}}\left(\tau_{1}, k_{1}\right)\right|\left|\widehat{v_{2}}\left(\tau_{2}, k_{2}\right)\right|\left|\widehat{v_{3}}\left(\tau_{3}, k_{3}\right)\right| d \tau_{1} d \tau_{2} d \tau_{3}
$$

where $\tau=\tau_{1}+\tau_{2}+\tau_{3}, k=k_{1}+k_{2}+k_{3}$ and

$$
A(N):=\left\{\left(k_{1}, k_{2}, k_{3}\right) \in \mathbb{Z}^{3},\left|k_{1}\right| \leq N / 2,\left|k_{2}\right| \leq N / 2 \text { and }\left|k_{3}\right|>N\right\} .
$$

To do this we will use the famous resonance relation for the Schrödinger equation. Setting $\sigma=\tau+k^{2}, \sigma_{1}=\tau_{1}+k_{1}^{2}, \sigma_{2}=\tau_{2}+k_{2}^{2}$ and $\tilde{\sigma}_{3}=\tau_{3}-k_{3}^{2}$, it holds

$$
\sigma-\sigma_{1}-\sigma_{2}-\tilde{\sigma}_{3}=2\left(k_{3}+k_{1}\right)\left(k_{3}+k_{2}\right) \text {. }
$$

This ensures that on $\mathbb{R}^{3} \times A(N)$,

$$
\max \left(|\sigma|,\left|\sigma_{1}\right|,\left|\sigma_{2}\right|,\left|\tilde{\sigma}_{3}\right|\right) \gtrsim N^{2}
$$

Therefore we get, thanks to (7) and (9),

$$
\begin{aligned}
J & \lesssim N^{-1 / 4+2 \epsilon} \int_{\mathbb{R}^{3}} \sum_{\left(k_{1}, k_{2}, k_{3}\right) \in A(N)}|\sigma|^{1 / 8-\epsilon}|\widehat{w}(\tau, k)|\left|\sigma_{1}\right|^{1 / 8}\left|\widehat{v_{1}}\left(\tau_{1}, k_{1}\right)\right| \\
& \left.\lesssim \sigma_{2}\right|^{1 / 8}\left|\widehat{v_{2}}\left(\tau_{2}, k_{2}\right)\right|\left|\tilde{\sigma}_{3}\right|^{1 / 8}\left|\widehat{v_{3}}\left(\tau_{3}, k_{3}\right)\right| d \tau_{1} d \tau_{2} d \tau_{3} \\
& \prod_{i=1}^{-1 / 4+2 \epsilon}\left\|\mathcal{F}^{-1}\left(|\sigma|^{1 / 8-\epsilon}|\widehat{w}|\right)\right\|_{L^{4}(\mathbb{R} \times \mathbb{T})}\left\|\mathcal{F}^{-1}\left(|\tilde{\sigma}|^{1 / 8}\left|\widehat{v_{3}}\right|\right)\right\|_{L^{4}(\mathbb{R} \times \mathbb{T})} \\
& \lesssim N^{-1 / 4+2 \epsilon}\|w\|_{X^{1 / 2-\epsilon, 0}} \prod_{i=1}^{3}\left\|v_{i}\right\|_{X^{1 / 2,0}}
\end{aligned}
$$

This completes the proof of the lemma. 
We are now in position to prove the Proposition 5.2. Let $u_{0} \in \mathcal{A}$ we decompose $u(t)=S(t) u_{0}$ by

$$
u(t)=v(t)+w(t)
$$

where $v$ and $w$ are defined as in (25)-(26). Note that (26) can be rewritten as

$$
w_{t}+i w_{x x}+\gamma w=-i Q_{N}\left(|u|^{2} u\right)+i Q_{N}\left(|v|^{2} v\right)
$$

which clearly ensures that (32) holds.

5.1.2. Decay in time of $w$. From Theorem 3.1, (11) and the fact that $u$ belongs to the attractor, we know that for all $t \in \mathbb{R}$ (recall that $\mathcal{A}$ is positively and negatively invariant by the flow),

$$
\|u\|_{X_{] t-1, t+1[}^{1 / 2+, 0}} \lesssim M_{0} \text { and }\left\|Q_{N} u\right\|_{X_{] t-1, t+1[}^{1 / 2,0}} \lesssim \varepsilon(N)
$$

Since $u \in X_{]-T, T[}^{1 / 2+, 0}$ for any $T>0$, proceeding as in Theorem 3.1 it is easy to prove that the Cauchy problem for $w$ is locally well-posed in $L^{2}(\mathbb{T})$ and thus $w \in$ $C\left([-\alpha, \alpha] ; L^{2}(\mathbb{T})\right)$ for some $\alpha>0$. Moreover, proceeding as in the proof of Theorem 3.1 , we get the following estimate on $w$ for all $t \in]-\alpha, \alpha[$ and $0<\delta<\min (\mid t-$ $\alpha|| t+,\alpha \mid)$,

$$
\|w\|_{X_{] t-\delta, t+\delta[}^{1 / 2+, 0}} \lesssim\|w(t)\|_{L^{2}(\mathbb{T})}+\delta^{0+}\|w\|_{X_{] t-\delta, t+\delta[}^{1 / 2+, 0}}\left(\|w\|_{X_{] t-\delta, t+\delta[}^{1 / 2+, 0}}^{2}+\|u\|_{X_{] t-\delta, t+\delta[}^{1 / 2+, 0}}^{2}+1\right) .
$$

Assuming that $\|w(t)\|_{L^{2}(\mathbb{T})}$ is bounded by some constant $A>0$ on $[0, T]$ for some positive time $T \in] 0, \alpha$, we deduce that there exists $\delta_{0}=\delta_{0}(A)>0$ such that for $0<\delta<\delta_{0}$ small enough,

$$
\|w\|_{X_{] t-\delta, t+\delta[}^{1 / 2+, 0}} \lesssim\|w(t)\|_{L^{2}(\mathbb{T})}, \quad \forall t \in[0, T] .
$$

From now on, we fix $0<\delta<\delta_{0}$ such that (35) holds. From this last inequality and (4) we infer that

$$
\inf _{\tau \in] t, t+\delta[}\|w(\tau)\|_{L^{2}(\mathbb{T})} \gtrsim\|w(t)\|_{L^{2}(\mathbb{T})}, \forall t \in[0, T] .
$$

Multiplying (26) with $2 \bar{w}$ and integrating over $\mathbb{T}$ we get

$$
\begin{aligned}
\frac{d}{d t}\|w\|_{L^{2}(\mathbb{T})}^{2}+2 \gamma\|w\|_{L^{2}(\mathbb{T})}^{2} \leq & 2\left|\int_{\mathbb{T}}\left(2|w|^{2} u-w^{2} \bar{u}\right) \bar{w}\right| \\
& +2\left|\int_{\mathbb{T}} u^{2} \bar{w}^{2}\right| .
\end{aligned}
$$

Integrating (37) with respect to time we obtain the following estimate for any $t \in[0, T]$

$$
\begin{aligned}
\|w(t+\delta)\|_{L^{2}(\mathbb{T})}^{2} \leq & \|w(t)\|_{L^{2}(\mathbb{T})}^{2} e^{-\gamma \delta}-\gamma \int_{t}^{t+\delta} e^{-\gamma(t+\delta-s)}\|w(s)\|_{L^{2}(\mathbb{T})}^{2} d s \\
& +2\left|\int_{t}^{t+\delta} e^{-\gamma(t+\delta-s)} \int_{\mathbb{T}}\left(2|w|^{2} u-w^{2} \bar{u}\right) \bar{w} d s\right| \\
& +2\left|\int_{t}^{t+\delta} e^{-\gamma(t+\delta-s)} \int_{\mathbb{T}} u^{2} \bar{w}^{2} d s\right|
\end{aligned}
$$


From (36) we infer that,

$$
-\gamma \int_{t}^{t+\delta} e^{-\gamma(t+\delta-s)}\|w(s)\|_{L^{2}(\mathbb{T})}^{2} d s \leq-C\left(1-e^{-\gamma \delta}\right)\|w(t)\|_{L^{2}(\mathbb{T})}^{2} .
$$

Let us estimate now the two last time integrals in (38). To do this we will extensively use that, following [11], for $h \in X_{] t, t+\delta[}^{-1 / 2+, \alpha}$ and $g \in X_{] t, t+\delta[}^{1 / 2+\alpha, 0}$ with $0<\alpha<<1$, it holds

(40) $\left|\int_{t}^{t+\delta} e^{-\gamma(t+\delta-s)} \int_{\mathbb{T}} h(s, x) \overline{g(s, x)} d x d s\right| \lesssim C(\delta, \alpha)\|h\|_{X_{] t, t+\delta[}^{-1 / 2+\alpha, 0}}\|g\|_{X_{] t, t+\delta[}^{1 / 2+\alpha, 0}}$.

Indeed, taking time extensions $\tilde{h}$ and $\tilde{g}$ of $h$ and $g$ such that $\|\tilde{g}\|_{X^{-1 / 2+\alpha, 0}} \leq$ $2\|g\|_{X_{] t, t+\delta[}^{-1 / 2+\alpha, 0}}$ and $\|\tilde{h}\|_{X^{1 / 2+\alpha, 0}} \leq 2\|h\|_{X_{] t, t+\delta[}^{1 / 2+\alpha, 0}}$, we have

$$
\begin{aligned}
& \left|\int_{t}^{t+\delta} e^{-\gamma(t+\delta-s)} \int_{\mathbb{T}} h(s, x) \overline{g(s, x)} d x d s\right| \\
= & \left|\int_{\mathbb{R}} \int_{\mathbb{T}} \tilde{h}(s, x) \chi_{[t, t+\delta]} e^{-\gamma(t+\delta-s)} \overline{\tilde{g}(s, x)} d x d s\right| \\
\lesssim & \|\tilde{h}\|_{X^{-1 / 2+\alpha, 0}}\left\|\chi_{[t, t+\delta]} e^{-\gamma(t+\delta-s)} \tilde{g}\right\|_{X^{1 / 2-\alpha, 0}}
\end{aligned}
$$

with

$$
\begin{aligned}
\left\|\chi_{[t, t+\delta]} e^{-\gamma(t+\delta-s)} \tilde{g}\right\|_{X^{1 / 2-\alpha, 0}} \lesssim & \left\|\chi_{[t, t+\delta]} e^{-\gamma(t+\delta-s)}\right\|_{L^{\infty}}\|\tilde{g}\|_{X^{1 / 2-\alpha, 0}} \\
& +\left\|\chi_{[t, t+\delta]} e^{-\gamma(t+\delta-s)}\right\|_{H^{1 / 2-\alpha}}\|\tilde{g}\|_{L^{\infty}\left(\mathbb{R} ; L^{2}(\mathbb{T})\right)} \\
\lesssim & C(\alpha, \delta)\|\tilde{g}\|_{X^{1 / 2+\alpha, 0}}
\end{aligned}
$$

With (40) at hand, we deduce from (10), (33) and (35) that

$$
\begin{aligned}
I_{1} & :=\left|\int_{t}^{t+\delta} e^{-\gamma(t+\delta-s)} \int_{\mathbb{T}}\left(2|w|^{2} u-w^{2} \bar{u}\right) \bar{w} d s\right| \\
& \lesssim\left\|\left(2|w|^{2} u-w^{2} \bar{u}\right)\right\|_{X_{] t, t+\delta[}^{-1 / 2+, 0}}\|w\|_{X_{] t, t+\delta[}^{1 / 2+,}} \\
& \lesssim\|w\|_{X_{] t, t+\delta[}^{1 / 2+, 0}}^{3}\left(\|w\|_{X_{] t, t+\delta[}^{1 / 2+, 0}}+\|u\|_{X_{] t, t+\delta[}^{1 / 2+, 0}}\right) \\
& \lesssim\|w(t)\|_{L^{2}(\mathbb{T})}^{3}\left(\|w(t)\|_{L^{2}(\mathbb{T})}+M_{0}\right)
\end{aligned}
$$

To estimate the last time integral we split it into two parts in the following way:

$$
\begin{aligned}
I_{2}:= & \left|\int_{t}^{t+\delta} e^{-\gamma(t+\delta-s)} \int_{\mathbb{T}} u^{2} \bar{w}^{2} d s\right| \\
= & \left|\int_{t}^{t+\delta} e^{-\gamma(t+\delta-s)} \int_{\mathbb{T}}\left(Q_{N / 2} u(s)\right)\left(Q_{N / 2} u(s)+2 P_{N / 2} u(s)\right)(\bar{w}(s))^{2} d s\right| \\
& +\left|\int_{t}^{t+\delta} e^{-\gamma(t+\delta-s)} \int_{\mathbb{T}}\left(P_{N / 2} u(s)\right)^{2}(\bar{w}(s))^{2} d s\right| \\
(42)= & I_{21}+I_{22} .
\end{aligned}
$$

To estimate $I_{21}$ we proceed as above and use (33) to get

$$
\begin{aligned}
I_{21} & \lesssim\|u\|_{X_{] t, t+\delta[}^{1 / 2+, 0}}\left\|Q_{N / 2} u\right\|_{X_{] t, t+\delta[}^{1 / 2+, 0}}\|w\|_{X_{] t, t+\delta[}^{1 / 2+, 0}}^{2} \\
& \lesssim M_{0} \varepsilon(N / 2)\|w(t)\|_{L^{2}(\mathbb{T})}^{2} .
\end{aligned}
$$


Finally, to estimate $I_{22}$ we use Lemma 5.4 (recall that $w=Q_{N} w$ ) and (35) to obtain

$$
\begin{aligned}
I_{22} & \lesssim\left\|\left(P_{N / 2} v(s)\right)^{2} \bar{w}\right\|_{X_{j t, t+\delta !}^{-1 / 2+, 0}}\|w\|_{X_{j t, t+\delta !}^{1 / 2+, 0}} \\
& \lesssim \frac{M_{0}^{2}}{N^{1 / 4-}}\|w\|_{X_{1 t, t+\delta \mid}^{1 / 2+, 0}}^{2} \\
& \lesssim \frac{M_{0}^{2}}{N^{1 / 4-}}\|w(t)\|_{L^{2}(\mathbb{T})}^{2} .
\end{aligned}
$$

Gathering (38)-(44) we thus infer that for all $t \in[0, T]$,

$$
\begin{aligned}
\|w(t+\delta)\|_{L^{2}(\mathbb{T})}^{2}- & \|w(t)\|_{L^{2}(\mathbb{T})}^{2} e^{-\gamma \delta} \\
\leq & {\left[C_{1}\left(\|w(t)\|_{L^{2}(\mathbb{T})}\left(\|w(t)\|_{L^{2}(\mathbb{T})}+M_{0}\right)+M_{0} \varepsilon(N / 2)+\frac{M_{0}^{2}}{N^{1 / 4-}}\right)\right.} \\
& \left.-C_{2}\left(1-e^{-\gamma \delta}\right)\right]\|w(t)\|_{L^{2}(\mathbb{T})}^{2} .
\end{aligned}
$$

Since $w(0)=Q_{N}\left(u_{0}\right)$, according to Proposition 5.3, we can choose $N>0$ large enough so that the right-hand side of the above inequality is negative at $t=0$. By direct iteration in time and (36) we thus infer that

$$
\|w(t)\|_{L^{2}(\mathbb{T})} \lesssim e^{-\gamma t}\|w(0)\|_{L^{2}(\mathbb{T})} \lesssim e^{-\gamma t}\left\|Q_{N} u_{0}\right\|_{L^{2}(\mathbb{T})} \lesssim e^{-\gamma t} \varepsilon(N), \forall t \in[0, T] .
$$

In particular, $\|w(t)\|_{L^{2}(\mathbb{T})}$ is bounded by $A=C\|w(0)\|_{L^{2}(\mathbb{T})}$ on $[0, T]$ and from the local well-posedness of $(26)$ we infer that $w \in C\left(\mathbb{R}_{+} ; L^{2}(\mathbb{T})\right)$ and that (46) holds actually for any $T>0$. This proves the first assertion of Proposition 5.2.

5.1.3. Estimate on $Q_{N} v$. First since $u=v+w$ we deduce from the preceding subsection that, for $N$ large enough, $v$ is well defined for all positive time and $v \in C\left(\mathbb{R}_{+} ; L^{2}(\mathbb{T})\right)$. Now, since by construction $P_{N} v=P_{N} u$ and $u$ belongs to the global attractor, we get thanks to (33) that

$$
\left\|P_{N} v(t)\right\|_{H^{2}} \lesssim\left\|P_{N} u(t)\right\|_{H^{2}} \leq C(N), \forall t \geq 0 .
$$

It thus remains to control the high frequencies of $v$. Inspired by $[\mathbf{2 1}]$ we introduce the functions $g$ and $g_{N}$ defined by

$$
\hat{g}(k):=\frac{\widehat{f}(k)}{-i k^{2}+\gamma} \text { and } g_{N}:=Q_{N} g
$$

so that $g_{N}$ satisfies the equation

$$
\partial_{t} g_{N}+i \partial_{x x} g_{N}+\gamma g_{N}=Q_{N} f .
$$

Therefore, setting $z:=Q_{N} v-g_{N}, z=Q_{N} z$ and is solution of

$$
\left\{\begin{array}{l}
z_{t}+i z_{x x}+\gamma z+i Q_{N}\left(|v|^{2} v\right)=0 \\
z(0)=-g_{N}
\end{array} .\right.
$$

We plan to prove that $z(t)$ is uniformly bounded in $H^{2}(\mathbb{T})$ for positive times. We will need the following result on the behavior of $g_{N}$ with respect to $N$.

Lemma 5.5. $g_{N} \in H^{2}(\mathbb{T}) \cap X_{]-1,1[}^{1 / 2,1}$ and it holds

$$
\left\|g_{N}\right\|_{H^{2}(\mathbb{T})}+\left\|g_{N}\right\|_{X_{]-1,1]}^{1 / 2,1}} \leq \varepsilon(N)
$$

where $\varepsilon(N) \rightarrow 0$ as $N \rightarrow+\infty$. 
Proof. It is clear that

$$
\left\|g_{N}\right\|_{H^{2}(\mathbb{T})} \leq\left\|Q_{N} f\right\|_{L^{2}(\mathbb{T})} \rightarrow 0 \text { as } N \rightarrow+\infty .
$$

Let now $\psi \in C_{0}^{\infty}(]-2,2[)$ such that $\psi \equiv 1$ on $[-1,1]$. It holds

$$
\begin{aligned}
\left\|g_{N}\right\|_{X_{]-1,1}^{1 / 2,1}} \leq & \left\|\psi g_{N}\right\|_{X^{1 / 2,1}}=\left\|\left\langle\tau+k^{2}\right\rangle^{1 / 2}\langle k\rangle \hat{\psi} \hat{g_{N}}\right\|_{L^{2}(\mathbb{R} \times \mathbb{Z})} \\
\leq & \left\|\langle\tau\rangle^{1 / 2} \hat{\psi}\right\|_{L^{2}(\mathbb{R})}\left\|\langle k\rangle \hat{g_{N}}\right\|_{L^{2}(\mathbb{Z})}+ \\
& \|\psi\|_{L^{2}(\mathbb{R})}\left\|\langle k\rangle^{2} \hat{g_{N}}\right\|_{L^{2}(\mathbb{Z})} \lesssim\left\|g_{N}\right\|_{H^{2}(\mathbb{T})} .
\end{aligned}
$$

This completes the proof of the lemma.

It is worth noticing that combining (29), (50), (33), (35) and (46), there exists $\delta_{0}>0$ such that

$$
\|z(t)\|_{L^{2}(\mathbb{T})} \leq \varepsilon(N) \text { and }\|z\|_{X_{] t-\delta_{0}, t+\delta_{0}[}^{1 / 2+,}} \lesssim M_{0}, \forall t \geq 0
$$

where $\varepsilon(N) \rightarrow 0$ as $N \rightarrow+\infty$. Therefore, taking $\beta>0$ small enough, it holds

$$
\|z\|_{X_{] t-\delta_{0}, t+\delta_{0}[}^{1 / 2,0}} \lesssim\|z\|_{X_{] t-\delta_{0}, t+\delta_{0}[}^{0,0}}^{\frac{2 \beta}{1+2 \beta}}\|z\|_{X_{] t-\delta_{0}, t+\delta_{0}[}^{1 / 2+\beta, 0}}^{\frac{1}{1+2 \beta}} \lesssim \varepsilon^{\prime}(N)
$$

where $\varepsilon^{\prime}(N) \rightarrow 0$ as $N \rightarrow+\infty$.

According to the linear estimates (5)-(6), to prove that the equation (49) is globally well-posed in $H^{2}(\mathbb{T})$, it suffices to prove the following estimate :

LEMma 5.6. Assuming that $z \in X_{I}^{1 / 2+, 2}$ for some time interval $I \subset \mathbb{R}$ with $|I| \leq 1$. The following estimate holds :

$$
\left\|Q_{N}\left(|v|^{2} v\right)\right\|_{X_{I}^{-1 / 2+\epsilon, 2}} \lesssim C(N)+\|z\|_{X_{I}^{1 / 2+, 2}}
$$

Proof. We decompose $v$ as $v=P_{N} u+z+g_{N}$ so that we have to estimate

$$
\left\|Q_{N}\left(\left|P_{N} u+z+g_{N}\right|^{2}\left(P_{N} u+z+g_{N}\right)\right)\right\|_{X_{I}^{-1 / 2+\epsilon, 2}} \text {. }
$$

Let us first estimate the expression containing $g_{N}$, i.e. terms of the form

$$
\left\|Q_{N}\left(\overline{g_{N}} w_{1} w_{2}\right)\right\|_{X_{I}^{-1 / 2+\epsilon, 2}} \text { or }\left\|Q_{N}\left(\bar{w}_{1} g_{N} w_{2}\right)\right\|_{X_{I}^{-1 / 2+\epsilon, 2}}
$$

with $\left(w_{1}, w_{2}\right) \in\left\{g_{N}, P_{N} u, z\right\}^{2}$. By the triangle inequality we can write

$$
\begin{aligned}
& \left\|Q_{N}\left(\overline{g_{N}} w_{1} w_{2}\right)\right\|_{X_{I}^{-1 / 2+\epsilon, 2}} \\
\leq & \left\|Q_{N}\left(\overline{g_{N}} w_{1} w_{2}\right)\right\|_{X_{I}^{-1 / 2+\epsilon, 0}}+\left\|Q_{N}\left(\overline{g_{N}} D_{x}^{2} \tilde{w}_{1} w_{2}\right)\right\|_{X_{I}^{-1 / 2+\epsilon, 0}} \\
& +\left\|Q_{N}\left(\overline{g_{N}} w_{1} D_{x}^{2} \tilde{w}_{2}\right)\right\|_{X_{I}^{-1 / 2+\epsilon, 0}}+\left\|Q_{N}\left(\overline{D_{x}^{2} g_{N}} w_{1} w_{2}\right)\right\|_{X_{I}^{-1 / 2+\epsilon, 0}} \\
& +\left\|Q_{N}\left(\overline{g_{N}} D_{x}^{2} g_{N} w_{2}\right)\right\|_{X_{I}^{-1 / 2+\epsilon, 0}}
\end{aligned}
$$

with $\left(\tilde{w}_{1}, \tilde{w}_{2}\right) \in\left\{P_{N} u, z\right\}$. The terms containing no derivative on $g_{N}$ of the above right-hand side can be estimated thanks to (7), (50) and (51) by

$$
\begin{aligned}
& \left\|g_{N}\right\|_{X_{I}^{1 / 2,0}}\left(\left\|P_{N} u\right\|_{X_{I}^{1 / 2,2}}+\|z\|_{X_{I}^{1 / 2,2}}+\right. \\
& \left.\left\|g_{N}\right\|_{X_{I}^{1 / 2,0}}\right)\left(\left\|P_{N} u\right\|_{X_{I}^{1 / 2,0}}+\|z\|_{X_{I}^{1 / 2,0}}+\left\|g_{N}\right\|_{X_{I}^{1 / 2,0}}\right) \\
& \quad \lesssim \varepsilon(N)\left(M_{0}^{2} N^{2}+M_{0}\|z\|_{X_{I}^{1 / 2,2}}+\varepsilon(N)\right) .
\end{aligned}
$$


For the terms that contains two derivatives on $g_{N}$ we write

$$
\begin{aligned}
& \left\|Q_{N}\left(D_{x}^{2} \overline{g_{N}} w_{1} w_{2}\right)\right\|_{X_{I}^{-1 / 2+\epsilon, 0}} \\
\lesssim & \left\|g_{N}\right\|_{H^{2}(\mathbb{T})}\left\|w_{1}\right\|_{L_{I}^{\infty} L^{\infty}(\mathbb{T})}\left\|w_{2}\right\|_{L_{I}^{\infty} L^{\infty}(\mathbb{T})} \\
\lesssim & \varepsilon(N)\left\|w_{1}\right\|_{L_{I}^{\infty} L^{2}(\mathbb{T})}^{3 / 4}\left\|w_{2}\right\|_{L_{t}^{\infty} L^{2}(\mathbb{T})}^{3 / 4}\left\|w_{1}\right\|_{L_{I}^{\infty} H^{2}(\mathbb{T})}^{1 / 4}\left\|w_{2}\right\|_{L_{I}^{\infty} H^{2}(\mathbb{T})}^{1 / 4} \\
\lesssim & \varepsilon(N)\left(M_{0}^{2} N^{2}+\varepsilon(N)\|z\|_{X_{I}^{1 / 2+, 2}}+\varepsilon(N)\right) .
\end{aligned}
$$

The terms of the form

$$
\left\|Q_{N}\left(\bar{w} g_{N} w_{2}\right)\right\|_{X_{I}^{-1 / 2+\epsilon, 2}}
$$

can be treated exactly in the same way. It remains to consider the terms where $g_{N}$ is not involved. From (7) and (52),

$$
\left\||z|^{2} z\right\|_{X_{I}^{-1 / 2+\epsilon, 2}} \lesssim\|z\|_{X_{I}^{1 / 2,0}}^{2}\|z\|_{X_{I}^{1 / 2,2}} \lesssim \varepsilon(N)^{2}\|z\|_{X_{I}^{1 / 2,2}}
$$

and

$$
\begin{aligned}
\left\|P_{N} u|z|^{2}\right\|_{X_{I}^{-1 / 2+\epsilon, 2}} & +\left\|\overline{P_{N} u} z^{2}\right\|_{X_{I}^{-1 / 2+\epsilon, 2}} \\
& \lesssim\left\|P_{N} u\right\|_{X_{I}^{1 / 2,0}}\|z\|_{X_{I}^{1 / 2,0}}\|z\|_{X_{I}^{1 / 2,2}}+\left\|P_{N} u\right\|_{X^{1 / 2,2}}\|z\|_{X^{1 / 2,0}}^{2} \\
& \lesssim \varepsilon(N) M_{0}\|z\|_{X_{I}^{1 / 2,0}}+N^{2} M_{0} \varepsilon(N) N^{-2}\|z\|_{X_{I}^{1 / 2,2}} \\
& \lesssim \varepsilon(N) M_{0}\|z\|_{X_{I}^{1 / 2,2}} .
\end{aligned}
$$

To deal with $\left(P_{N} u\right)^{2} \bar{z}$ we decompose it as

$$
\left(P_{N} u\right)^{2} \bar{z}=\left(Q_{N / 2} P_{N} u\right)\left(Q_{N / 2} P_{N} u+2 P_{N / 2} u\right) \bar{z}+\left(P_{N / 2} u\right)^{2} \bar{z}:=A_{1}+A_{2} .
$$

Clearly, (33) yields

$$
\left\|A_{1}\right\|_{X_{I}^{-1 / 2+\epsilon, 2}} \lesssim \varepsilon(N / 2) M_{0}\|z\|_{X_{I}^{1 / 2,2}}
$$

and using Lemma 5.4 it is easy to check that

$$
\left\|A_{2}\right\|_{X^{-1 / 2+\epsilon, 2}} \lesssim N^{-1 / 4} M_{0}^{2}\|z\|_{X_{I}^{1 / 2,2}}
$$

Finally,

$$
\begin{aligned}
\left\|\left|P_{N} u\right|^{2} z \mid\right\|_{X^{-1 / 2+\epsilon, 2}} & \lesssim\left\|P_{N} u\right\|_{X_{I}^{1 / 2,0}}\left\|P_{N} u\right\|_{X_{I}^{1 / 2,2}}\|z\|_{X_{I}^{1 / 2,0}}+\left\|P_{N} u\right\|_{X_{I}^{1 / 2,0}}^{2}\|z\|_{X_{I}^{1 / 2,2}} \\
& \lesssim M_{0}^{2}\left(N^{2} N^{-2}+1\right)\|z\|_{X_{I}^{1 / 2,2}} \lesssim M_{0}^{2}\|z\|_{X_{I}^{1 / 2,2}}
\end{aligned}
$$

and

$$
\left\|\left|P_{N} u\right|^{2} P_{N} u \mid\right\|_{X_{I}^{-1 / 2+\epsilon, 2}} \lesssim N^{2} M_{0}^{3}
$$

Gathering all the above estimates, (53) follows.

From the above lemma and (5)-(6) we deduce that $z \in X_{] t-\delta, t+\delta[}^{1 / 2+, 2}$ for any $t \geq 0$ and any $0<\delta<\delta_{0}$. Moreover, it holds

$$
\|z\|_{X_{] t-\delta, t+\delta[}^{1 / 2+, 2}} \lesssim\|z(t)\|_{H^{2}(\mathbb{T})}+C \delta^{0+}\left(C(N)+\|z\|_{X_{] t-\delta, t+\delta[}^{1 / 2+, 2}}\right) .
$$

This ensures that for $\delta_{0}>0$ small enough,

$$
\|z\|_{X_{] t-\delta_{0}, t+\delta_{0}[}^{1 / 2+, 2}} \lesssim\|z(t)\|_{H^{2}(\mathbb{T})}+C(N) .
$$


We will proceed as in the preceding subsection. From now on we fix $0<\delta<\delta_{0}$ such that (64) holds. As in (36), this implies that

$$
\inf _{\tau \in] t-\delta, t+\delta[}\|z(\tau)\|_{H^{2}(\mathbb{T})} \geq C\|z(t)\|_{H^{2}(\mathbb{T})}-C(N), \forall t \geq 0 .
$$

On the other hand, taking the real part of the $H^{2}(\mathbb{T})$ hermitian-product of $(49)$ with $2 z$, we get

$$
\frac{d}{d t}\|z\|_{H^{2}(\mathbb{T})}^{2}+2 \gamma\|z\|_{H^{2}(\mathbb{T})}^{2}=\Re\left[-i 2\left(\left(Q_{N}\left(|v|^{2} v\right), z\right)\right)_{H^{2}}\right] .
$$

Integrating with respect to time this implies the following estimate for any $t \geq 0$,

$$
\begin{aligned}
\|z(t+\delta)\|_{H^{2}(\mathbb{T}) \leq}^{2} & \|z(t)\|_{H^{2}(\mathbb{T})}^{2} e^{-\gamma \delta}-\gamma \int_{t}^{t+\delta} e^{-\gamma(t+\delta-s)}\|z(s)\|_{H^{2}(\mathbb{T})}^{2} d s \\
& +2\left[\int_{t}^{t+\delta} e^{-\gamma(t+\delta-s)} \Im\left[\left(\left(Q_{N}\left(|v(s)|^{2} v(s)\right), z(s)\right)\right)_{H^{2}}\right] d s\right. \\
\leq & \|z(t)\|_{H^{2}(\mathbb{T})}^{2} e^{-\gamma \delta}+\left(1-e^{-\gamma \delta}\right)\left(C(N)-C\|z(t)\|_{H^{2}(\mathbb{T})}^{2}\right) \\
& +2\left|\Im\left[\int_{t}^{t+\delta} e^{-\gamma(t+\delta-s)}\left(\left(|v(s)|^{2} v(s), z(s)\right)\right)_{H^{2}} d s\right]\right|
\end{aligned}
$$

To estimate the last term of the above right-hand side we decompose $v$ as in Lemma 5.6. In view of (40) and (54)-(62) to get the following estimate :

$$
\left|\Im\left[\int_{t}^{t+\delta} e^{-\gamma(t+\delta-s)}\left(\left(|v(s)|^{2} v(s), z(s)\right)\right)_{H^{2}} d s\right]\right| \lesssim C(N)+\varepsilon(N)\|z\|_{X^{1 / 2+, 2}}^{2},
$$

we only have to care about

$$
I:=\mid \int_{t}^{t+\delta} e^{-\gamma(t+\delta-s)} \Im\left(\left(\left|P_{N} u(s)\right|^{2} z(s), z(s)\right)_{H^{2}} d s \mid .\right.
$$

To deal with this term we decompose $\Im\left(\left(\left|P_{N} u(s)\right|^{2} z(s), z(s)\right)\right)_{H^{2}}$ as

$$
\begin{aligned}
\Im\left(\left(\left|P_{N} u\right|^{2} z, z\right)\right)_{H^{1}} d s & +\Im \int_{\mathbb{T}}\left(2 \partial_{x}\left(\left|P_{N} u\right|^{2}\right) \partial_{x} z+\partial_{x}^{2}\left(\left|P_{N} u\right|^{2}\right) z\right) \partial_{x}^{2} \bar{z} \\
& +\Im \int_{\mathbb{T}}\left|P_{N} u\right|^{2}\left|\partial_{x}^{2} z\right|^{2} d x
\end{aligned}
$$

and notice that the last term vanishes. We thus get thanks to (40) and (10),

$$
\begin{aligned}
I \lesssim & \left\|\left|P_{N} u\right|^{2} z\right\|_{X_{] t-\delta, t+\delta[}^{-1 / 2+, 1}}\|z\|_{X_{] t-\delta, t+\delta[}^{1 / 2+, 1}} \\
& +\left\|2 \partial_{x}\left(\left|P_{N} u\right|^{2}\right) \partial_{x} z+\partial_{x}^{2}\left(\left|P_{N} u\right|^{2}\right) z\right\|_{X_{] t-\delta, t+\delta[}^{-1 / 2+, 0}}\|z\|_{X_{] t-\delta, t+\delta[}^{1 / 2+, 2}} \\
\lesssim & M_{0}^{2} \varepsilon(N)\|z\|_{X_{] t-\delta, t+\delta[}^{1 / 2+2}}+ \\
& \left(M_{0}^{2} N \varepsilon(N)^{1 / 2}\|z\|_{X_{] t-\delta, t+\delta[}^{1 / 2+, 2}}^{1 / 2}+M_{0}^{2} N^{2} \varepsilon(N)\right)\|z\|_{X_{] t-\delta, t+\delta[}^{1 / 2+, 2}} \\
\lesssim & \varepsilon(N)\|z\|_{X_{] t-\delta, t+\delta[}^{1 / 2+, 2}}^{2}+C(N) .
\end{aligned}
$$

Combining this last estimate with (55)-(62), (68) follows. 
We thus infer that

$$
\begin{aligned}
\|z(t+\delta)\|_{H^{2}(\mathbb{T})}^{2} \leq & \|z(t)\|_{H^{2}(\mathbb{T})}^{2} e^{-\gamma \delta}+C(N) \\
& +C_{1}\left(\varepsilon(N)-C_{2}\left(1-e^{-\gamma \delta}\right)\right)\|z(t)\|_{H^{2}(\mathbb{T})}^{2} .
\end{aligned}
$$

For $N$ large enough the last term of the right-hand side is clearly negative and is bounded from above by

$$
-\alpha\|z(t)\|_{H^{2}(\mathbb{T})}^{2}
$$

for some small real number $\alpha>0$. This ensures that, taking $N>0$ large enoug, there exists $C(N)>0$ such that

$$
\|z(t)\|_{H^{2}(\mathbb{T})} \leq C(N), \forall t \geq 0,
$$

and thus on account of (47), (50), (64) and the definition of $z$, there exists $K(N)>0$ such that

$$
\|v(t)\|_{H^{2}(\mathbb{T})} \leq K(N), \forall t \geq 0 .
$$

This completes the proof of Proposition 5.2.

5.2. Compactness in $H^{2}(\mathbb{T})$. To prove the compactness in $H^{2}(\mathbb{T})$, it suffices to show that

$$
\|z(t)\|_{H^{2}(\mathbb{T})} \leq \varepsilon(N), \forall t \geq 0 .
$$

Indeed, this will imply the same estimate on $v$ and thus on any $a \in \mathcal{A}$ which will clearly prove the $H^{2}(\mathbb{T})$ compactness of $\mathcal{A}$. For proving (74), we revisit Lemma 5.6 with (28) at hand. It is then easy to check that the terms involving $g_{N}$ in Lemma 5.6 (see (55)-(56)) can now be controlled by

$\left\|g_{N}\right\|_{H^{2}(\mathbb{T})}\left(\left\|g_{N}\right\|_{H^{2}(\mathbb{T})}^{2}+\left\|P_{N} u\right\|_{L^{\infty}\left(I ; H^{2}(\mathbb{T})\right)}^{2}+\|z\|_{L^{\infty}\left(I ; H^{2}(\mathbb{T})\right)}^{2}\right) \lesssim \varepsilon(N)\left(\|z\|_{X_{I}^{1 / 2+, 2}+1}\right)$. and that (see (69) above)

$$
\begin{aligned}
& \left|\Im \int_{t}^{t+\delta} e^{-\gamma(t+\delta-s)}\left(\left|P_{N} u(s)\right|^{2} z(s), z(s)\right)_{H^{2}(\mathbb{T})} d s\right| \\
& \quad \lesssim\left\|\left|P_{N} u\right|^{2} z\right\|_{L^{\infty}(] t, t+\delta\left[; H^{1}(\mathbb{T})\right)}\|z\|_{L^{\infty}(] t, t+\delta\left[; H^{1}(\mathbb{T})\right)} \\
& \quad+\left(\left\|\partial_{x}\left(\left|P_{N} u\right|^{2}\right) \partial_{x} z\right\|_{L^{\infty}(] t, t+\delta\left[; L^{2}(\mathbb{T})\right)}+\right. \\
& \left.\quad\left\|\partial_{x}^{2}\left(\left|P_{N} u\right|^{2}\right) z\right\|_{L^{\infty}(] t, t+\delta\left[; L^{2}(\mathbb{T})\right)}\right)\|z\|_{L^{\infty}(] t, t+\delta\left[; H^{2}(\mathbb{T})\right)} \\
& \quad \lesssim\left\|P_{N} u\right\|_{L^{\infty}(] t, t+\delta\left[; H^{2}(\mathbb{T})\right)}^{2}\left(N^{-2}+N^{-1}\right)\|z\|_{L^{\infty}(] t, t+\delta\left[; H^{2}(\mathbb{T})\right)}^{2} \\
& \quad \lesssim N^{-1}\|z\|_{X_{] t, t+\delta[}^{1 / 2+, 2}} .
\end{aligned}
$$

To conclude we need the following estimate that we will prove hereafter.

$$
\left\|Q_{N}\left(\left|P_{N} u\right|^{2} P_{N} u\right)\right\|_{X_{] t-1, t+1[}^{-1 / 2+, 2}} \lesssim \varepsilon(N), \forall t \geq 0 .
$$

Proceeding as in the derivation of (68) but with(75)-(77) at hand it is now easy to see that it actually holds

$$
\left.\mid \Im\left[\int_{t}^{t+\delta} e^{-\gamma(t+\delta-s)}\left(Q_{N}\left(|v(s)|^{2} v(s)\right), z(s)\right)\right)_{H^{2}} d s\right] \mid \lesssim \varepsilon(N)\left(1+\|z\|_{X_{] t, t+\delta[}^{1 / 2+, 2}}^{2}\right)
$$


and thus

$$
\begin{aligned}
\|z(t+\delta)\|_{H^{2}(\mathbb{T})} \leq & \|z(t)\|_{H^{2}(\mathbb{T})} e^{-\gamma \delta}+\varepsilon(N) \\
& +C_{1}\left(\varepsilon(N)-C_{2}\left(1-e^{-\gamma \delta}\right)\right)\|z(t)\|_{H^{2}(\mathbb{T})}^{2}
\end{aligned}
$$

which proves (74).

5.2.1. Proof of Estimate (77). Note first that due to the frequency projections it clearly holds

$$
\begin{aligned}
\left\|Q_{N}\left(\left|P_{N} u\right|^{2} P_{N} u\right)\right\|_{X_{|t, t+\delta|}^{-1 / 2+2}} \lesssim & \left\|Q_{N}\left(\left|P_{N} u\right|^{2} P_{N} Q_{N / 3} u\right)\right\|_{X_{1 t, t+\delta \delta}^{-1 / 2+, 2}} \\
& +\left\|Q_{N}\left(\left(P_{N} u\right)^{2} \overline{P_{N} Q_{N / 3} u}\right)\right\|_{X_{j t, t+\delta \mid}^{-1 / 2+, 2}}
\end{aligned}
$$

Therefore, on account of (28) it is easy to check that we have only to care about

$$
\left\|Q_{N}\left(\left|P_{N} u\right|^{2} \partial_{x}^{2} P_{N} Q_{N / 3} u\right)\right\|_{X_{] t-1, t+1[}^{-1 / 2+, 0}}+\left\|Q_{N}\left(\left(P_{N} u\right)^{2} \overline{\partial_{x}^{2} P_{N} Q_{N / 3} u}\right)\right\|_{X_{] t-1, t+1[}^{-1 / 2+, 0}}
$$

since (77) is obvious for terms that involved less that two derivatives on $Q_{N / 3} u$.

To bound (79) we will use that there exist $C>0$ such that for all $t \geq 0$, $u-g \in X_{t t-1, t+1[}^{1 / 2+, 2}$ (see (48) for the definition of $g$ ) with

$$
\|u-g\|_{X_{t t-1, t+1[}^{1 / 2+, 2}} \leq C .
$$

Indeed from (72) and (64) we know that for $N_{0}>0$ large enough there exists $\delta>0$ and $C\left(N_{0}\right)>0$ such that

$$
\left\|Q_{N_{0}}(v-g)\right\|_{X_{] t-\delta, t+\delta[}^{1 / 2+, 2}}=\|z\|_{X_{] t-\delta, t+\delta[}^{1 / 2+, 2}} \leq C\left(N_{0}\right), \quad \forall t \geq 0
$$

and thus $v-g$ is bounded in $X_{] t-\delta, t+\delta[}^{1 / 2+, 2}$ uniformly in $t \geq 0$. Now, proceeding as in the proof of (28) we can decompose $u$ as $u=v_{n}+w_{n}$ with $\left\|w_{n}\right\|_{L^{\infty}(]-1,+\infty\left[; L^{2}(\mathbb{T})\right)} \rightarrow 0$ as $n \rightarrow \infty$ and $\left\|v_{n}-g\right\|_{X_{j t-1, t+1[}^{1 / 2+, 2}} \leq C$ for all $t \geq 0$. We thus infer that

$$
w_{n} \rightarrow 0 \text { weakly star in } L^{\infty}(] t-1, t+1\left[; L^{2}(\mathbb{T})\right)
$$

and thus

$$
v_{n}-g \rightarrow u-g \text { in } X_{] t-1, t+1[}^{1 / 2+, 2}
$$

which proves (80).

Now, on account of Lemma 5.5, it clearly holds

$$
\begin{gathered}
\left\|Q_{N}\left(\left|P_{N} u\right|^{2} \partial_{x}^{2} P_{N} Q_{N / 3} g\right)\right\|_{X_{] t-1, t+1[}^{-1 / 2+, 0}}+\left\|Q_{N}\left(\left(P_{N} u\right)^{2} \overline{\partial_{x}^{2} P_{N} Q_{N / 3} g}\right)\right\|_{X_{] t-1, t+1[}^{-1 / 2+, 0}} \\
\lesssim\left\|Q_{N / 3} g\right\|_{H^{2}}\left\|P_{N} u\right\|_{L^{\infty}\left(\mathbb{R}_{+} ; H^{2}(\mathbb{T})\right)}^{2} \lesssim \varepsilon(N) .
\end{gathered}
$$

It thus remains to estimate

$$
\begin{aligned}
& \left\|Q_{N}\left(\left|P_{N} u\right|^{2} \partial_{x}^{2} P_{N} Q_{N / 3}(u-g)\right)\right\|_{X_{] t-1, t+1[}^{-1 / 2+, 0}} \\
& +\left\|Q_{N}\left(\left(P_{N} u\right)^{2} \overline{\partial_{x}^{2} P_{N} Q_{N / 3}(u-g)}\right)\right\|_{X_{] t-1, t+1[}^{-1 / 2+, 0}}
\end{aligned}
$$


We take extensions $\theta=P_{N} \theta$ of $P_{N} u$ and $\vartheta=Q_{N / 3} P_{N} \vartheta$ of $Q_{N / 3} P_{N}(u-g)$ such that $\|\theta\|_{X^{1 / 2,0}} \leq 2\left\|P_{N} u\right\|_{X_{\mid t-1, t+1[}^{1 / 2,0}}$ and $\|\vartheta\|_{X^{1 / 2,2}} \leq 2\left\|Q_{N / 3} P_{N} \vartheta\right\|_{X_{\mid t-1, t+1[}^{1 / 2,2}}$. By duality it suffices to prove that for $\epsilon>0$ small enough,

$\sup _{\|h\|_{X^{1 / 2-\epsilon, 0}=1}}\left[\left|\left(h, Q_{N}\left(|\theta|^{2} \partial_{x}^{2} \vartheta\right)\right)_{L^{2}}\right|+\left|\left(h, Q_{N}\left(\theta^{2} \partial_{x}^{2} \bar{\vartheta}\right)\right)_{L^{2}}\right|\right] \lesssim \varepsilon(N)\|\theta\|_{X^{1 / 2,0}}^{2}\|\vartheta\|_{X^{1 / 2,2}}$ and thus to estimate

$$
\begin{aligned}
J:= & \int_{\mathbb{R}^{3}} \sum_{\left(k_{1}, k_{2}, k_{3}\right) \in A(N)}|\widehat{h}(\tau, k)|\left|\widehat{\theta}\left(\tau_{1}, k_{1}\right)\right|\left|\widehat{\bar{\theta}}\left(\tau_{2}, k_{2}\right)\right|\left|k_{3}\right|^{2}\left|\widehat{\vartheta}\left(\tau_{3}, k_{3}\right)\right| d \tau_{1} d \tau_{2} d \tau_{3} \\
& +\int_{\mathbb{R}^{3}} \sum_{\left(k_{1}, k_{2}, k_{3}\right) \in A(N)}|\widehat{h}(\tau, k)|\left|\widehat{\theta}\left(\tau_{1}, k_{1}\right)\right|\left|\widehat{\theta}\left(\tau_{2}, k_{2}\right)\right|\left|k_{3}\right|^{2}\left|\widehat{\bar{\vartheta}}\left(\tau_{3}, k_{3}\right)\right| d \tau_{1} d \tau_{2} d \tau_{3}
\end{aligned}
$$

where $\tau=\tau_{1}+\tau_{2}+\tau_{3}, k=k_{1}+k_{2}+k_{3}$ and

$$
\begin{aligned}
& A(N):=\left\{\left(k_{1}, k_{2}, k_{3}\right) \in \mathbb{Z}^{3},\left|k_{i}\right| \leq N\right. \\
& \text { for } \left.i \in\{1,2,3\},\left|k_{3}\right|>N / 3, N<\left|k_{1}+k_{2}+k_{3}\right| \leq 3 N\right\} .
\end{aligned}
$$

On $\mathbb{R}^{3} \times A(N)$, the resonance relation (31) clearly yields

$$
\max \left(|\sigma|,\left|\sigma_{1}\right|,\left|\sigma_{2}\right|,\left|\tilde{\sigma}_{3}\right|\right) \gtrsim\left|\left(k_{1}+k_{3}\right)\left(k_{2}+k_{3}\right)\right| \gtrsim N^{2},
$$

where $\sigma=\tau+k^{2}, \sigma_{1}=\tau_{1}+k_{1}^{2}, \sigma_{2}=\tau_{2}+k_{2}^{2}$ and $\tilde{\sigma}_{3}=\tau_{3}-k_{3}^{2}$. Moreover, noticing that $k_{1}+k_{2} \neq 0$ on $A(N)$, we infer that

$$
\max \left(|\sigma|,\left|\sigma_{1}\right|,\left|\tilde{\sigma}_{2}\right|,\left|\sigma_{3}\right|\right) \gtrsim\left|\left(k_{1}+k_{2}\right)\left(k_{3}+k_{2}\right)\right| \gtrsim N,
$$

where $\tilde{\sigma}_{2}=\tau_{2}-k_{2}^{2}$ and $\sigma_{3}=\tau_{3}+k_{3}^{2}$. Therefore proceeding as in the proof of Lemma 5.4 we obtain

$$
\begin{aligned}
J & \lesssim N^{-1 / 8+\epsilon}\|h\|_{X^{1 / 2-\epsilon, 0}} \prod_{i=1}^{3}\|\theta\|_{X^{1 / 2,0}}^{2}\|\vartheta\|_{X^{1 / 2-, 2}} \\
& \lesssim C M_{0}^{2} N^{-1 / 8+\epsilon}\|h\|_{X^{1 / 2-\epsilon, 0}}
\end{aligned}
$$

which completes the proof of (77).

\section{References}

[1] N. Akroune, Regularity of the attractor for a weakly damped Schrödinger equation on $\mathbb{R}$, Applied Math. Letters, 12, (1999), 45-48

[2] J. Ball, Global attractors for damped semilinear wave equations. Partial differential equations and applications, Discrete Contin. Dyn. Syst. 10 (2004), no. 1-2, 31-52.

[3] J. Bourgain, Fourier transform restriction phenomena for certain lattice subsets and application to nonlinear evolution equations I. The Schrödinger equation, GAFA, 3 (1993), 157-178.

[4] M. Christ, Power series solution of a nonlinear Schrödinger equation. 131-155, Ann. of Math. Stud., 163, Princeton University Press, Princeton, NJ, 2007.

[5] M. Christ, J. Colliander and T. Tao, Instability of the periodic nonlinear Schrödinger equation, preprint arXiv:math/0311048.

[6] J.-M. Ghidaglia, Finite dimensional behavior for the weakly damped driven Schrödinger equations, Ann. Inst. Henri Poincaré, 5, (1988), 365-405.

[7] J. Ginibre, Le problème de Cauchy pour des EDP semi-linéaires périodiques en variables d'espace (d'après Bourgain), in Séminaire Bourbaki 796, Astérique 237, 1995, 163-187.

[8] J. Ginibre, Y. Tsutsumi and G. Velo, On the Cauchy problem for the Zakharov system, J. Funct. Analysis 133 (1995), 50-68. 
[9] O. Goubet, Regularity of the attractor for the weakly damped nonlinear Schrödinger equations, Applicable Anal., 60, (1996), 99-119.

[10] O. Goubet, Asymptotic smoothing effect for weakly damped Korteweg-de Vries equations, Discrete Contin. Dyn. Syst. 6 (2000), 625-644.

[11] O. Goubet and R. Rosa, Asymptotic smoothing and the global attractor of a weakly damped $K d V$ equation on the real line J. Differ. Equa. (2002), 53.

[12] O. Goubet and L. Molinet, Global attractor for weakly damped nonlinear Schrödinger equations in $L^{2}(\mathbb{R})$, to appear in Nonlinear Analysis, TMA.

[13] A. Grünrock and S. Herr, Low regularity local well-posedness of the derivative nonlinear Schrödinger equation with periodic initial data, SIAM J. Math. Anal. 39 (2008), 1890-1920.

[14] J. Hale, Asymptotic behavior of Dissipative Systems, Math. surveys and Monographs, vol 25, AMS, Providence, 1988.

[15] A. Miranville and S. Zelik, Attractors for dissipative partial differential equations in bounded and unbounded domains, Handbook of Differential Equations, Evolutionary Partial Differential Equations, C.M. Dafermos and M. Pokorny eds., Elsevier, Amsterdam, to appear

[16] I. Moise, R. Rosa and X. Wang, Attractors for non-compact semigroups via energy equations, Nonlinearity 11, (1998), no. 5, 1369-1393.

[17] L. Molinet, Global well-posedness in the energy space for the Benjamin-Ono equation on the circle, Math. Ann. 337 (2007), 353-383.

[18] L. Molinet, On ill-posedness for the one-dimensional periodic cubic Schrodinger equation, To appear in Math. Research Letters (2008).

[19] G. Raugel, Global attractors in partial differential equations. Handbook of dynamical systems, Vol. 2, 885-982, North-Holland, Amsterdam, 2002.

[20] R. Temam, Infinite Dimensional Dynamical Systems in Mechanics and Physics, SpringerVerlag, Second Edition, 1997.

[21] K. Tsugawa, Global well-posedness for the KdV equations on the real line with low regularity forcing terms. Commun. Contemp. Math. 8(5) (2006), pp. 681-713.

[22] X. Wang, An energy equation for the weakly damped driven nonlinear Schrödinger equations and its applications to their attractors, Physica D, 88, (1995), 167-175.

L.A.G.A., Institut Galilée, Université Paris-Nord, 93430 Villetaneuse, France

E-mail address: molinet@math.univ-paris13.fr 